\title{
The Effect of Concurrent Tetanus-diphtheria Vaccination on the Antibody Response to Rabies Vaccine: A Preliminary Study
}

\author{
Hasan Tahsin Gozdas ${ }^{1}$, Oguz Karabay ${ }^{1}$, Aziz Ogutlu ${ }^{1}$, Ertugrul Guclu ${ }^{1}$, \\ Yusuf Yurumez ${ }^{2}$, Mehmet Koroglu ${ }^{3}$, Unal Erkorkmaz ${ }^{4}$ \\ ${ }^{1}$ Department of Infectious Diseases and Clinical Microbiology, Sakarya \\ University Faculty of Medicine, Sakarya, Turkey; \\ ${ }^{2}$ Department of Emergency Medicine, Sakarya University Faculty of Medicine, \\ Sakarya, Turkey; \\ ${ }^{3}$ Department of Medical Microbiology, Sakarya University Faculty of Medicine, \\ Sakarya, Turkey; \\ ${ }^{4}$ Department of Biostatistics, Sakarya University Faculty of Medicine, Sakarya, \\ Turkey
}

Received January 14, 2018; Accepted October 5, 2018.

Key words: Rabies vaccine - Tetanus-diphtheria vaccine - Rabies antibody Antibody response

\begin{abstract}
The number of studies in the literature investigating the effect of tetanus vaccination on rabies prophylaxis is rather limited. In this study, we aimed to investigate the effect of concurrent tetanus-diphtheria (Td) vaccination on the antibody response to rabies vaccine. The data of consecutive 80 patients who presented to Sakarya University Training and Research Hospital, Department of Emergency due to rabies suspected exposure between 15 October 2012 and 12 June 2013 were enrolled to this study. Postexposure rabies prophylaxis had been given to all cases, however concurrent tetanus vaccination had been administered to some of them according to their need. Cases were divided into two parts according to their receipt of tetanus prophylaxis as rabies only group (group $R, n=37$ ), and rabies and tetanus-diphtheria group (group $R+T d, n=43$ ). Rabies antibody levels were tested in sera of the cases at first and postvaccination $21^{\text {st }}$ day. The median antibody levels of each group were measured and compared with each other statistically. In our study, postvaccination $21^{\text {st }}$ day antibody level of
\end{abstract}

This study was supported by Sakarya University BAP (project number: 2013-80-02-001).

Mailing Address: Dr. Hasan Tahsin Gozdas, Department of Infectious Diseases and Clinical Microbiology, Sakarya University Faculty of Medicine, Sakarya, Turkey; Phone: +902 642552 110; Fax: +902 642552 102;

e-mail: dr.htgozdas@yahoo.com.tr 
group $R$ was $0.68 \mathrm{IU} / \mathrm{ml}$ (IQR: 0.79 ), while the same for group $\mathrm{R}+\mathrm{Td}$ was $0.52 \mathrm{IU} / \mathrm{ml}$ (IQR: 0.48) $(p=0.022)$. Concurrent administration of $T d$ vaccine was found to have a significant negative effect on the antibody response to rabies vaccine. Our results should be confirmed with further studies including more cases.

\section{Introduction}

According to the World Health Organization (WHO) data, about 60,000 people die due to rabies and more than 10 million people expose to rabies-suspected bites annually worldwide. Prophylaxis is the major priority due to the fatal outcome of the disease. Post-exposure prophylaxis (PEP) has a very important place due to life saving effect (Coleman, 2004; Shead et al., 2009; World Health Organization, 2013). In post-exposure rabies prophylaxis (PERP), four doses of vaccination administered on day 0, 3, 7 and 14 is known to provide adequate seroprotection against rabies (Manning et al., 2008; http://www.cdc.gov/rabies/resources/acip_recommendations .html).

Antibody response to rabies vaccine can be influenced by many factors such as individual's age, immunologic condition and body mass index (BMI) (Johnson et al., 2010; Banga et al., 2014). To our knowledge, there are a limited number of studies regarding the effect of simultaneous tetanus-diphtheria $(\mathrm{Td})$ vaccination on rabies prophylaxis. The aim of the present study was to investigate the effect of simultaneous $\mathrm{Td}$ vaccination on the antibody response to rabies vaccine.

\section{Material and Methods}

In this study, retrospective data records of 80 consecutive patients who were admitted to the Department of Emergency of 400-bed tertiary care hospital of Sakarya University and given PEP between 15 October 2012 and 12 June 2013 were analysed and rabies antibody levels in previously stored serum samples were studied.

\section{Ethical consent}

Ethical consent was obtained from the Ethics Committee of Sakarya University Faculty of Medicine. Ethics committee approval number: B.30.2.S AÜ.0.20.05.04.050.01.04/41.

\section{Inclusion and exclusion criteria}

The inclusion criteria:

- Having a record in the Department of Emergency because of rabies suspected exposure in the study period and vaccinated with at least three doses of rabies vaccine

The exclusion criteria:

- Receiving immunosuppressive therapy

- Having immunosuppressive disease (e.g. AIDS) 


\section{- Being pregnant}

- Having incomplete data record

Although 92 patients were included in our study initially, 12 patients were excluded due to various reasons after applying the exclusion criteria. The study was completed with the remaining 80 patients (Figure 1).

\section{Study design}

In this retrospective study, rabies suspected bites were considered as dirty wounds. Patients who received PERP were also screened for tetanus prophylaxis, so they were divided into two groups according to the simultaneous administration of tetanus prophylaxis. These groups were called as the rabies only group (group $R$, $n=37$ ), and the rabies and tetanus-diphtheria group (group $R+T d, n=43$ ). At the beginning of the study, it was planned to test rabies antibodies at admission and day 21. So, serum collection was prospectively done for rabies PEP cases and then these samples were retrospectively analysed for the effect of Td vaccine.

\section{Administration of vaccines}

- Purified inactivated rabies vaccine prepared on Vero cells had been administered intramuscularly to both group $\mathrm{R}$ and group $\mathrm{R}+\mathrm{Td}$ (Abhayrab $2.5 \mathrm{IU} / 0.5 \mathrm{ml}$ containing human serum albumin, maltose, thiomersal and phosphate buffer).

- Adsorbed diphtheria-tetanus vaccine with aluminium adjuvant was administered intramuscularly to group $\mathrm{R}+\mathrm{Td}$ simultaneously from the opposite upper limb than that of the rabies vaccine (Td-VAC $0.5 \mathrm{ml}$ including aluminium phosphate, thiomersal, sodium acetate and sodium chloride). Diphtheria-tetanus vaccine had not been administered to some of the patients who had received tetanus prophylaxis within the last five years (Advisory Committee on Immunization Practices, 2012).

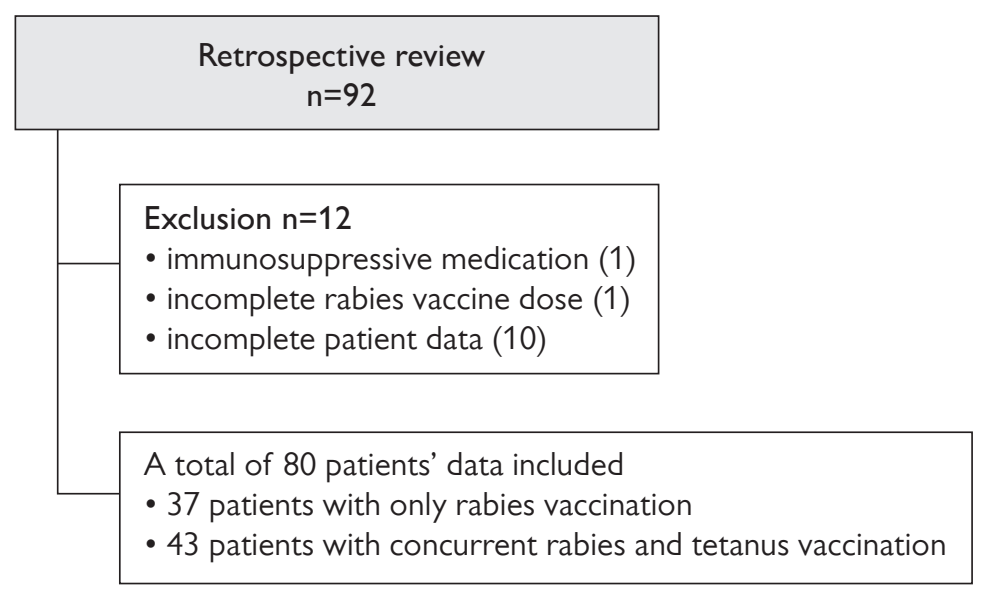

Figure 1 - Flow chart of the study. 
- Additionally, immunoglobulin had been administered into and around the wounds to the patients as necessary according to the guidelines (Talan et al., 1999).

- Rabies prophylaxis had been administered by Department of Emergency nurses after skin disinfection with $70 \%$ alcohol.

\section{Recording patient data}

Demographic characteristics of the patients, locations and sizes of the bites, category of exposure, number of rabies vaccine dose, whether simultaneous tetanus prophylaxis was administered or not, BMl, smoking, comorbid diseases, species of the biting animal, vaccination status of the biting animal, post-exposure admission period, whether there was a history of rabies or tetanus prophylaxis within the last five years or not and allergy history were obtained from patient data.

\section{Blood sampling and storage}

Five $\mathrm{ml}$ of patient serum obtained at admission and day 21 had been stored in a freezer $\left(-20^{\circ} \mathrm{C}\right)$ until the study day. On the study day, serum samples were thawed at room temperature. Rabies antibody levels in these samples were measured quantitatively by microELISA method using Platelia Rabies II kit (Biorad, France) in a full automatic microELISA device (Triturus, Grifols, Spain).

\section{Statistical methods}

When continuous variables showing normal distributions were compared between the two groups, independent two sample $t$-test was used and when continuous variables not showing normal distribution was compared within the same group, Mann-Whitney $U$ test was used. Continuous variables were shown with mean value and standard error, otherwise median value and interquartile range $(\mathrm{IQR}=$ third quartile - first quartile). When categorical variables were compared between the two groups, Pearson's chi-square test was used. Categorical variables were shown with number and percent. Multiple linear regression analysis was performed to determine the factors affecting postvaccination $21^{\text {st }}$ day rabies antibody level. A $\mathrm{p}$-value of less than 0.05 was considered statistically significant. Statistical analysis was performed by a statistics software (IBM SPSS Statistics 20, SPSS Inc., IBM Co., Somers, NY).

\section{Results}

Patients

In our study, group R consisted of 37 patients (26 males, 11 females) and the median age in this group was 13 (IQR: 37 ). Group R+Td consisted of 43 patients (24 males, 19 females) and the median age in this group was 37 (IQR: 37 ). 53 (66.3\%) patients had category II exposure, 27 patients (33.8\%) had category III exposure. 


\section{Demographic characteristics}

50 patients $(62.5 \%)$ were male and 30 patients $(37.5 \%)$ were female. The mean age was $32.9 \pm 21.9$. The average surface area of the bite was $5.3 \pm 11.7 \mathrm{~cm}^{2}$. Mean body weight was $61.7 \pm 21.9 \mathrm{~kg}$, the mean height was $1.6 \pm 0.2 \mathrm{~m}$ and the mean BMI was $23.5 \pm 5.7 \mathrm{~kg} / \mathrm{m}^{2}$. In total, 53 cases had category II exposure and 27 patients had category III exposure. So, 52 patients in our study had received 3 doses $(0,3,7)$ and 28 patients had received 4 doses of rabies vaccine $(0,3,7,14)$.

In group $R$, average surface area of the bite was $6.3 \pm 3.15 \mathrm{~cm}^{2}$. The mean body weight was $51 \pm 23 \mathrm{~kg}$, the mean height was $1.49 \pm 0.22 \mathrm{~m}$, and the mean BMl was $21.52 \pm 5.71 \mathrm{~kg} / \mathrm{m}^{2}$. Although eight patients $(21.6 \%)$ in group R had comorbid disease, no patient had diabetes mellitus (DM). In this group, 25 (67.6\%) patients had category II exposure and 12 (32.4\%) had category III exposure. So, 26 patients had received 3 doses and 11 patients had received 4 doses of rabies vaccine.

In group $R+T d$, average surface area of the bite was $4.37 \pm 7.33 \mathrm{~cm}^{2}$, and the mean body weight was $71 \pm 15 \mathrm{~kg}$, mean height was $1.7 \pm 0.1 \mathrm{~m}$ and the mean BMI was $25.2 \pm 5.2 \mathrm{~kg} / \mathrm{m}^{2}$. Eight patients in this group (18.6\%) had comorbid disease and three of them had DM. In this group, $28(65.1 \%)$ patients had category II exposure, 15 (34.9\%) had category III exposure. So, 26 patients received 3 doses and 17 patients received 4 doses of rabies vaccine.

In our study, the lower limit of the detection of rabies antibody level was $0.12 \mathrm{IU} / \mathrm{ml}$ and the upper limit for the same was $4 \mathrm{IU} / \mathrm{ml}$. Values below and above these could not be determined. When the median antibody levels of the groups were measured, it was seen that postvaccination $21^{\text {st }}$ day antibody level of group $R$ was $0.68 \mathrm{IU} / \mathrm{ml}$ (IQR: 0.79 ), while the same for group $\mathrm{R}+\mathrm{Td}$ was $0.52 \mathrm{IU} / \mathrm{ml}$ (IQR: 0.48) $(p=0.022)$.

For defining obesity, a BMI z-score of $\geq 2$ was used for patients under 18 years of age (Inokuchi et al., 2011) and a BMI of $\geq 30 \mathrm{~kg} / \mathrm{m}^{2}$ was used for patients over 18 years of age (World Health Organization, 2006). According to this definition, four patients in group $R$ and eight patients in group $R+T d$ were defined as obese.

\section{Table 1 - Comparison of each group in itself according to seroconvertion rate}

\begin{tabular}{lcccccc}
\hline & \multicolumn{3}{c}{ Group R } & \multicolumn{3}{c}{ Group R+Td } \\
\cline { 2 - 7 } & $<0.5(\mathrm{n}=9)$ & $\geq 0.5(\mathrm{n}=28)$ & $\mathrm{P}$-value & $<0.5(\mathrm{n}=21)$ & $\geq 0.5(\mathrm{n}=22)$ & P-value \\
\hline Age & $10(11.5)$ & $18.5(39.25)$ & 0.065 & $37(40)$ & $38.5(31.5)$ & 0.855 \\
\hline $\begin{array}{l}\text { Rabies } \\
\text { vaccine dose }\end{array}$ & $3(1)$ & $3(2)$ & 0.576 & $3(2)$ & $3(2)$ & 0.422 \\
\hline Obesity & 0 & $4(14.3)$ & 0.230 & $3(14.3)$ & $5(22.7)$ & 0.477 \\
\hline
\end{tabular}

Group R - rabies only group; Group R+Td - rabies and tetanus-diphtheria group 
Table 2 - Factors affecting postvaccination $21^{\text {st }}$ day rabies antibody level

\begin{tabular}{lcccc}
\hline & $\beta$ & $\mathrm{SE}$ & $95 \% \mathrm{Cl}$ & P-value \\
\hline Concurrent tetanus-diphtheria vaccination & -0.255 & 0.103 & -0.460 to -0.049 & 0.016 \\
Rabies vaccine dose (three vs. four) & -0.016 & 0.053 & -0.122 to 0.091 & 0.766 \\
Gender & -0.010 & 0.109 & -0.208 to 0.228 & 0.927 \\
Obesity & 0.088 & 0.145 & -0.202 to 0.378 & 0.546 \\
Smoking & 0.048 & 0.125 & -0.201 to 0.296 & 0.704 \\
Comorbidity & 0.025 & 0.100 & -0.174 to 0.224 & 0.804 \\
Age & 0.001 & 0.003 & -0.004 to 0.006 & 0.564 \\
\hline
\end{tabular}

$\beta$ - regression coefficient; SE - standard error; $\mathrm{Cl}$ - confidence interval

Each group was separated in itself into two parts based on seroconvertion rate and comparison of the groups was shown in Table 1.

The values equal to or above $0.5 \mathrm{IU} / \mathrm{ml}$ are universally considered as protective antibody response to rabies vaccination (World Health Organization, 2013). Hence, the critical limit of protective antibody level was accepted to be $\geq 0.5 \mathrm{IU} / \mathrm{ml}$ at day 21 postvaccination. Based on the multiple linear regression analysis, factors which can affect the logarithm of postvaccination $21^{\text {st }}$ day rabies antibody titers were presented in Table 2. According to this, simultaneous Td vaccination was determined as an independent factor which inversely affects rabies antibody response.

\section{Discussion}

Rabies and tetanus (in some countries Td) vaccines are often administered together. These different immunological products may have various effects on each other when administered simultaneously (Léry et al., 1986; Phanuphak et al., 1989; Moore et al., 2006). Effects of the tetanus vaccine on rabies vaccination have not been studied enough up to date. In a previous study investigating this interaction, rabies antibody response was found significantly higher when rabies vaccine was given in the same syringe with tetanus toxoid (Phanuphak et al., 1989). This was linked with the aluminium hydroxide in the tetanus vaccine which served as adjuvant for the rabies vaccine. In that study, purified Vero cell rabies vaccine and pure tetanus toxoid containing aluminium adjuvant were used. We used the same rabies vaccine [purified Vero cell rabies vaccine (Abhayrab $2.5 \mathrm{lU} / 0.5 \mathrm{ml}$ )], but a different tetanus vaccine [adsorbed diphtheria-tetanus vaccine with aluminium adjuvant (Td-VAC $0.5 \mathrm{ml}$ )] in our study. According to our results, simultaneous Td vaccination decreases the logarithm of postvaccination $21^{\text {st }}$ day rabies antibody levels. We concluded that simultaneously administered $\mathrm{Td}$ vaccine had a significant negative effect on the rabies antibody response $(p=0.016,95 \% \mathrm{Cl}=$ from -0.460 to -0.049$)$. Another important result was the fact that there was no significant difference between antibody levels obtained with three or four doses of rabies vaccine $(p=0.766,95 \% \mathrm{Cl}=$ from -0.122 to 0.091$)$. 
Immunologically, vaccines are classified as T-cell-dependent (e.g. rabies, tetanus, diphtheria, hepatitis B) and T-cell-independent (e.g. typhoid and pneumococcus) (Lang et al., 1997). Evaluating Td vaccine as an inversely affecting factor of rabies antibody levels, we believe that there may be a competitive inhibition between rabies antigens and $\mathrm{Td}$ toxoids since both stimulate $\mathrm{T}$-dependent antibody response. Therefore, it can be possible that $\mathrm{Td}$ vaccine might have a negative effect on rabies antibody level. In a study conducted on infants by Lang et al. (1997), effects of simultaneous DTP-IPV (combined diphtheria, pertussis, diphtheria-tetanus, and inactivated polio vaccine) and PVRV (purified Vero cell rabies vaccine) vaccines were investigated. Eighty four infants of two-month-old were included in that study. Diphtheria immunogenicity at first month was found lower in simultaneously PVRV and DTP-IPV administered group than that of only DTP-IPV administered group. This was linked with the competition between rabies antigens and other antigens. Similarly, we believe that competitive inhibition might have affected our results.

Antibody response against vaccination may be influenced by many factors. Age, smoking and obesity are the best known factors. In vaccine antibody studies related to hepatitis $B$ vaccination, the effects of these factors have been clearly demonstrated. Age, smoking and obesity negatively affect antibody response to hepatitis B vaccination (Ingardia et al., 1999; Sunbul et al., 2000; Mast and Ward, 2008). Age and genetic factors were previously reported to have a negative effect on the antibody response to rabies vaccination (Ceddia et al., 1982; Mastroeni et al., 1994; Kennedy et al., 2007).

Age may affect rabies vaccine antibody response. Some studies have reported a relationship between age and efficacy of rabies vaccine (Mastroeni et al., 1994; Kennedy et al., 2007; Morris et al., 2007; Robertson et al., 2010). However, age had no effect in our study $(p=0.564,95 \% \mathrm{Cl}=$ from -0.004 to 0.006$)$. This might be caused by the fact that most of our cases composed of young population. The mean age of our patients was $32.9 \pm 21$.9. It is well known that immune response decreases with advanced age. Indeed, many studies have reported the inverse effect between age and vaccine response (Mastroeni et al., 1994; Kennedy et al., 2007; Morris et al., 2007). We think, our results need to be confirmed with further studies including older age groups.

In our study, rabies antibody levels were only tested at day 21 after vaccination. Of our patients, 52 (65\%) had received three doses of rabies vaccine, while $28(35 \%)$ had four doses of rabies vaccine. In a previous study, all of the subjects receiving either three or four doses of rabies vaccine were found to have adequate seroconvertion (Robertson et al., 2010). Similarly, no significant effect of rabies vaccine dose (either three or four) on the antibody response was detected in our study $(p=-0.016,95 \% \mathrm{Cl}=$ from -0.122 to 0.091$)$. We think, this finding is valuable. If this result that we have found with a limited number of patients can be confirmed with larger study samples and if it could be possible to show that three doses of rabies vaccine was as effective as four doses by prospective studies, 
then it will be possible to increase the vaccine adherence and decrease vaccination outcomes. Therefore, we suggest that further studies need to be conducted to compare the efficacy of three and four doses of rabies vaccination.

Before concluding, we need to discuss the limitations of our study. The major limitation in this study is the low number of patients. In our study, one of the groups consisted of 37 patients while the other group consisted of 43 patients. In addition, a large portion of group R+Td consisted of pediatric patients who had received $\mathrm{Td}$ prophylaxis within the last five years. The median age of this group was lower than that of group R. Groups would be more homogenous if we had more patients. Another important limitation was the single measurement of antibody level after vaccination. Our patients' rabies antibody levels were tested postvaccination only at day 21 . It could be possible to show that the antibody levels rise above $0.5 \mathrm{IU} / \mathrm{ml}$ in more patients if it would be measured at a later date.

\section{Conclusion}

In this study on 80 patients who received PERP, we found that rabies antibody levels measured postvaccination at day 21 was not affected from age, gender, obesity, smoking, comorbid disease and number of rabies vaccine doses, but affected from simultaneously administered Td vaccine. In addition, there was no significant difference among antibody titres obtained with three or four doses of rabies vaccine. The results of our study should be confirmed with further studies including more cases.

Abstract form of this manuscript was presented at the $6^{\text {th }}$ EACID Congress 2014, Serbia.

\section{References}

Advisory Committee on Immunization Practices (2012) Recommended adult immunization schedule: United States. Ann. Intern. Med. 156, 211-217.

Banga, N., Guss, P., Banga, A., Rosenman, K. D. (2014) Incidence and variables associated with inadequate antibody titers after pre-exposure rabies vaccination among veterinary medical students. Vaccine $\mathbf{3 2}$, 979-983.

Ceddia, T., Natellis, C., Zigrino, A. G. (1982) Antibody response to rabies vaccine prepared in tissue culture of human diploid cells and inactivated, evaluated in different classes of age. Ann. Sclavo 24, 491-495.

Coleman, P. G. (2004) Estimating the public health impact of rabies. Emerg. Infect. Dis. 10, 140-142.

Ingardia, C. J., Kelley, L., Steinfeld, J. D., Wax, J. R. (1999) Hepatitis B vaccination in pregnancy: factors influencing efficacy. Obstet. Gynecol. 93, 983-986.

Inokuchi, M., Matsuo, N., Takayama, J. I., Hasegawa, T. (2011) BMI z-score is the optimal measure of annual adiposity change in elementary school children. Ann. Hum. Biol. 38, 747-751.

Johnson, N., Cunningham, A. F., Fooks, A. R. (2010) The immune response to rabies virus infection and vaccination. Vaccine 28, 3896-3901.

Kennedy, L. J., Lunt, M., Barnes, A., McElhinney, L., Fooks, A. R., Baxter, D. N., Ollier, W. E. (2007) Factors influencing the antibody response of dogs vaccinated against rabies. Vaccine 25, 8500-8507.

Lang, J., Duong, G. H., Nguyen, V. G., Le, T. T., Nguyen, C.V., Kesmedjian, V., Plotkin, S. A. (1997) Randomised feasibility trial of pre-exposure rabies vaccination with DTP-IPV in infants. Lancet 349, 1663-1665. 
Léry, L., Rotivel, Y., Trabaud, M. A., Parvaz, P., Mouterde, S., Relyveld, E. H. (1986) Combined tetanus-rabies vaccination. Dev. Biol. Stand. 65, 209-220.

Manning, S. E., Rupprecht, C. E., Fishbein, D., Hanlon, C. A., Lumlertdacha, B., Guerra, M., Meltzer, M. I., Dhankhar, P., Vaidya, S. A., Jenkins, S. R., Sun, B., Hull, H. F.; Advisory Committee on Immunization Practices Centers for Disease Control and Prevention (CDC) (2008) Human rabies prevention United States, 2008: recommendations of the Advisory Committee on Immunization Practices. MMWR Recomm. Rep. 57(RR-3), 1-28.

Mast, E., Ward, J. (2008) Hepatitis B vaccines. In: Vaccines, $5^{\text {th }}$ Edition, eds. Plotkin, S. A., Orenstein, W. A., Offit, P. A., pp. 205-242, Elsevier, Philadelphia.

Mastroeni, I., Vescia, N., Pompa, M. G., Cattaruzza, M. S., Marini, G. P., Fara, G. M. (1994) Immune response of the elderly to rabies vaccine. Vaccine 12, 518-520.

Moore, S. E., Collinson, A. C., Fulford, A. J., Jalil, F., Siegrist, C. A., Goldblatt, D., Hanson, L. A., Prentice, A. M. (2006) Effect of month of vaccine administration on antibody responses in The Gambia and Pakistan. Trop. Med. Int. Health 11, 1529-1541.

Morris, J., Crowcroft, N. S., Fook, A. R., Brookes, S. M., Andrews, N. (2007) Rabies antibody levels in bat handlers in the United Kingdom: Immune responses before and after purified chick embryo cell rabies booster vaccination. Hum. Vaccin. 3, 165-170.

Phanuphak, P., Khaoplod, P., Sriwanthana, B., Phanpanich, T., Wongurai, S., Roumiantzeff, M. (1989) Immunoenhancement with combined rabies and aluminium-adjuvanted tetanus vaccines. Vaccine $\mathbf{7}$, 249-252.

Robertson, K., Recuenco, S., Niezgoda, M., Garcia, E. J., Rupprecht, C. E. (2010) Seroconversion following incomplete human rabies postexposure prophylaxis. Vaccine 28, 6523-6526.

Shead, S., Wood, H., Artsob, H. (2009) Antibody response to rabies vaccine in healthy adults following primary immunization and the importance of occupational health surveillance programs. Int. J. Antimicrob. Agents 34, S5 (Suppl. 2).

Sunbul, M., Leblebicioglu, H., Esen, S., Eroglu, C., Barut, S. (2000) Response to hepatitis B vaccine in HBsAg/ anti-HBs negative and anti-HBc positive subjects. Scand. J. Infect. Dis. 32, 315-316.

Talan, D. A., Citron, D. M., Abrahamian, F. M., Moran, G. J., Goldstein, E. J. (1999) Bacteriologic analysis of infected dog and cat bites. Emergency Medicine Animal Bite Infection Study Group. N. Engl. J. Med. 340, 85-92.

World Health Organization (2006) World Health Organization Global Database on Body Mass Index. Geneva, Switzerland.

World Health Organization (2013) Rabies Vaccines. WHO Expert Consultation on Rabies. Second Report. WHO Technical Report Series. WHO Press, World Health Organization, Geneva. 